\title{
Apogossypolone, a small-molecule inhibitor of Bcl-2, induces radiosensitization of nasopharyngeal carcinoma cells by stimulating autophagy
}

\author{
JIN-HUA HE ${ }^{1,2}$, XIAO-LI LIAO ${ }^{2,3}$, WEI WANG $^{3}$, DAN-DAN LI ${ }^{2}$, WEN-DAN CHEN ${ }^{2}$, RONG DENG ${ }^{2}$, \\ DAJUN YANG ${ }^{4}$, ZE-PING HAN $^{1}$, JIAN-WEI JIANG ${ }^{3}$ and XIAO-FENG ZHU ${ }^{2}$
}

\begin{abstract}
${ }^{1}$ Department of Laboratory, Central Hospital of Panyu, Guangzhou; ${ }^{2}$ State Key Laboratory of Oncology in South China, Sun Yat-sen University Cancer Center, Guangzhou; ${ }^{3}$ Department of Biochemistry, Medical College, Jinan University, Guangzhou, Guangdong, P.R. China; ${ }^{4}$ Ascenta Therapeutics Incorporation, Malvern, PA 19355, USA
\end{abstract}

Received February 24, 2014; Accepted April 29, 2014

DOI: 10.3892/ijo.2014.2497

\begin{abstract}
Nasopharyngeal carcinoma (NPC) is a major cause of cancer deaths. Concurrent administration of radiation and chemotherapy is the treatment of choice for advanced NPC. Previously, we showed that apogossypolone (ApoG2) induced apoptosis by blocking the binding of Bcl-2 to Bax, arresting the cell cycle in the $\mathrm{S}$ phase, in turn inhibiting proliferation of NPC cells both in vitro and in vivo. In the present study, we showed that ApoG2 inhibited the proliferation of NPC cells in a dose-dependent manner. We treated CNE1, CNE2 and SUNE1 cells with ApoG2 for $72 \mathrm{~h}$, and calculated the $\mathrm{IC}_{50}$ values as $2.84,5.64$ and $2.18 \mu \mathrm{M}$, respectively. Normal NP69 cell proliferation was not significantly inhibited. ApoG2 treatment induced significant autophagy, demonstrated by an increase in LC3-II protein expression, reduced protein p62 expression, and accumulation of punctuate GFP-LC3 in the cytoplasm of CNE1 or CNE2 cells. Sh-Atg5 attenuated the autophagy induced by ApoG2, indicating that Atg5 was required for ApoG2-induced autophagy. In addition, ApoG2 treatment blocked the binding of Bcl-2 to Beclin 1 protein, releasing pro-autophagic Beclin 1, which in turn triggered the autophagic cascade. Colony formation assays indicated
\end{abstract}

Correspondence to: Professor Jian-Wei Jiang, Department of Biochemistry, Medical College, Jinan University, Guangzhou, Guangdong 510632, P.R. China

E-mail:jjw703@163.com

Professor Xiao-Feng Zhu, State Key Laboratory of Oncology in South China, Sun Yat-sen University Cancer Center, 651 Dongfeng East Road, Guangzhou, Guangdong 510060, P.R. China

E-mail: zhuxfeng@mail.sysu.edu.cn

Abbreviations: NPC, nasopharyngeal carcinoma; ApoG2, apogossypolone; $\mathrm{BH} 3$, Bcl-2-homology domain 3; DMSO, dimethylsulfoxide; $\mathrm{IC}_{50}, 50 \%$ inhibitory concentration; GFP-LC3, LC3 fused to green fluorescent protein

Key words: Bcl-2, nasopharyngeal carcinoma, autophagy, radiation that ApoG2 enhanced radiosensitization of CNE2 cells. In the ApoG2-plus-radiation combination group, more ring-shaped structures were evident in CNE1 and CNE2 cultures. LC3-II expression was enhanced and that of p62 reduced, compared to the ApoG2-only, radiation-only and control groups. ApoG2 enhanced the radiosensitivity of CNE2 xenografts in nude mice as measured by (C-T)/C ratios (as percentages); the values for the ApoG2 and radiation groups were $46.89 \%$ and $19.34 \%$, respectively. The ApoG2-plus-radiation group exhibited greater antitumor activity (the inhibitory rate was 61.64\%). Immunohistological staining showed that LC3-II expression became gradually upregulated in the ApoG2-plus-radiation group. Together, the results suggest that ApoG2 inhibits the binding of Bcl-2 to Beclin 1, inducing autophagy and radiosensitizing NPC cells both in vitro and in vivo.

\section{Introduction}

Nasopharyngeal carcinoma (NPC) is a human squamous cell carcinoma arising in the epithelium lining the upper region of the retronasal cavity. NPC has a remarkable geographic distribution, being very prevalent in southern China, Southeast Asia, and North Africa (1). The annual incidence rate is $\sim 30$ per 100,000 in such regions, thus 50-fold higher than in the Western world. Radiotherapy is the standard treatment for NPC, although such therapy is accompanied by undesirable complications. The overall 5-year survival rate is $\sim 60 \%$, and is even higher if intensity-modulated radiation therapy is employed $(2,3)$. Chemotherapy is important to control distant metastasis of chemoradiosensitive NPC, and thus plays an important role. However, neither an optimal combination of antitumor drugs, nor an ideal chemoradiotherapeutic regimen, has been well-established (4).

BCL-2 proteins are critical in terms of cell survival and are overexpressed in many tumors. To date, 25 members of the Bcl-2 family have been identified. The family is subdivided into three main groups based on differences in regions of the Bcl-2 homology (BH) domain, and the functions of these regions. One group of proteins inhibits apoptosis and exhibits four distinct $\mathrm{BH}$ domains; these proteins include $\mathrm{Bcl}-2, \mathrm{Bcl}-\mathrm{xL}$, 
Bcl-w, Mcl-1, Bcl-B and A1. The pro-apoptotic proteins are divided into two distinct groups. These are the multidomain proteins (Bax, Bak and Bok) containing three $\mathrm{BH}$ domains; and the (Bcl-2-homology domain 3-) BH3-only proteins (Bad, Bid, Bim, Bmf, Bik, Hrk, Noxa and Puma), which have a conserved $\mathrm{BH} 3$ domain that can bind to anti-apoptotic Bcl-2 proteins to promote apoptosis (5-7).

Anti-apoptotic Bcl-2 family proteins (Bcl-2, Bcl-xL, and Mcl-1) are commonly highly expressed in many types of cancer (8). Lu et al detected Bcl-2 in most (80\%) samples of undifferentiated NPC9 cells (9). Fan et al found that the expression of Bcl-2 protein was significantly higher in NPC tissues than in normal noncancerous nasopharyngeal epithelia (NPE) and hyperplastic NPE1 (10). Sheu et al immunohistochemically analyzed $\mathrm{Bcl}-2$ expression levels in biopsy specimens from 101 cases of NPC. The proportions of NPCs (80\%) and adjacent dysplastic lesions (71\%) expressing Bcl-2 were significantly higher than those of adjacent NPE (37\%) and the NPE of patients with chronic inflammation of the nasopharynx (30\%) (11). Yu et al explored whether Bcl-2 was overexpressed in NPCs at the time of diagnosis. The prevalence of Bcl-2 positivity in our material was $61 \%$ (31/51). Expression of Bcl-2 in NPCs is significantly associated with prognosis. Few patients with $\mathrm{Bcl}-2^{+}$tumors survive for 5 years; most develop local recurrences or distant metastases (12). Overexpression of anti-apoptotic proteins (Bcl-2, $\mathrm{Bcl}-\mathrm{XL}, \mathrm{Bcl}-\mathrm{w}$ and $\mathrm{Mcl}-1)$ reduces the pro-apoptotic response and causes NPC cells to develop resistance to traditional radiation and chemical therapies (13).

Targeting of the anti-apoptotic Bcl-2 family of proteins improves apoptosis and autophagy, thus overcoming drug resistance developing during cancer chemotherapy (14-16). Several groups have developed strategies to block the antiapoptotic activities of proteins of the Bcl-2 family. These feature the use of a Bcl-2 antisense oligodeoxynucleotide, peptides, and small-molecule inhibitors (17,18). As Bcl-2, Bcl-xL and Mcl-1 are critical regulators of apoptosis, being important anti-apoptotic molecules, it may be predicted that pan-inhibition of such Bcl-2 family members by small-molecule inhibitors would effectively induce cancer cell apoptosis. A Bcl-2 antisense oligodeoxynucleotide, oblimersen sodium (G3139, Genasense) has shown promise when used as an anti-apoptotic agent in tumor therapy, but G3139 targets only Bcl-2 mRNA, thus not mRNAs encoding Mcl-1 and Bcl-xl, which are also overexpressed in many cancer tissues $(5,7)$.

ABT-737 (A-779024, Abbott Laboratories) is a small molecule that targets anti-apoptotic Bcl-2 family proteins (Bcl-2, Bcl-XL and Bcl-w), thereby sequestering pro-apoptotic proteins with the $\mathrm{BH} 3$ domain, in turn promoting oligomerization of Bax and Bak and, ultimately, programmed cell death of malignant cells (18-21). ABT-737 is a potent small-molecule mimic of BH3 with high affinity Bcl-2, Bcl-xL and Bcl-w, but low affinity for Mcl-1 and $\mathrm{A} 1(\mathrm{Ki} \leq 1 \mathrm{nM}$ for Bcl-2, Bcl-XL and Bcl-w; Ki=0.46 nM for Bcl-B, Mcl-1 and Bfl1/A-1) (19). Consistent with the low affinity of ABT-737 for Mcl-1, several reports have suggested that high basal levels of Mcl-1 are associated with resistance to ABT-737. Combinations of ABT-737 with a cyclin-dependent kinase inhibitor (flavopiridol), arsenic trioxide, or fenretinide, are therapeutically synergistic, because the latter agent inactivates Mcl-1. Such work is paving the way for development of combination chemotherapies targeting the Bcl-2 family of proteins (22-25).

Gossypol is the first known compound to inhibit the Bcl-2, Bcl-XL and Mcl-1. Gossypol is a potentially toxic phenolic pigment found in the seed, stem, and root of the cotton plant, and was initially (during the 1950s) identified as an antifertility agent in China. Natural gossypol is a racemic mixture, and levo-gossypol (AT-101, Ascenta) phase II clinical trials are ongoing (in combination with rituximab) in chronic lymphocyte leukemia (CLL) patients and (in combination with docetaxel) in patients with hormone-refractory prostate cancer. In a fluorescence polarization-based binding assay, (-)-gossypol bound to Bcl-2, Bcl-xL, and Mcl-1 with Ki values of 320, 480 and $180 \mathrm{nM}$, respectively. Gastrointestinal toxicity was dose-limiting in a phase I/II clinical trial in prostate cancer patients $(26,27)$.

Apogossypolone (ApoG2) is a promising semi-synthetic derivative of gossypol, formed by removal of aldehyde groups, and binds to Bcl-2 family proteins with $\mathrm{Ki}$ values of 35 and $25 \mathrm{nM}$ for Bcl-2 and Mcl-1, respectively, and a $\mathrm{Ki}$ of $660 \mathrm{nM}$ for Bcl-XL $(13,18)$. ApoG2 exhibits a higher antitumor activity and lower toxicity than gossypol and other derivatives (29-31). Combinations of radiotherapy and chemotherapy have become standard treatments for NPC. We previously showed that ApoG2 indeed induced apoptosis by blocking the anti-apoptotic functions of Bcl-2 family members and suppressed tumor growth in NPC xenografts (13). ApoG2 disturbed the proliferation of NPC cells by suppressing c-Myc signaling and inducing arrest at the DNA synthesis stage in a large proportion of such cells (32), and suppressed growth of the human lymphoma cell line U937 by inhibiting the actions of anti-apoptotic proteins of the Bcl-2 family, and inducing mitochondrion-dependent apoptotic cell death (30). In the present study, we further investigated the radiosensitizing effects of ApoG2 on NPC cells.

\section{Materials and methods}

Cell culture and reagents. Human NPC cell line CNE1 (a highly differentiated line), the NPC, CNE2 and SUNE1 cell lines (which are poorly differentiated), and the NP69 normal human nasopharyngeal epithelial cell line, were cultured in RPMI-1640 with $10 \%$ (v/v) fresh bovine serum, $1 \mathrm{U} / \mathrm{ml}$ penicillin, and $1 \mathrm{mg} / \mathrm{ml}$ streptomycin at $37^{\circ} \mathrm{C}$ under $5 \%$ (v/v) $\mathrm{CO}_{2}$. ApoG2 was provided by Dr Dajun Yang, dissolved to $20 \mathrm{mM}$ in dimethylsulfoxide (DMSO), and stored at $-20^{\circ} \mathrm{C}$. The primary antibodies used for immunoblots and immunoprecipitations were anti-Bcl-2; anti-LC3; anti-GAPDH; anti-p62; and antiBeclin 1 (Cell Signaling, Danvers, MA, USA).

MTT assay. Cell viability was measured using the MTT assay. Mitochondria converted MTT from a soluble tetrazolium salt to an insoluble colored formazan precipitate, which was dissolved in DMSO and spectrophotometrically quantitated. NPC cells were plated in 96-well tissue culture plates (Costar, Cambridge, MA, USA) at a density of $8 \times 10^{4}$ cells $/ \mathrm{ml}$. The ApoG2 stock solution was serially diluted. All experiments were performed in duplicate or triplicate. MTT (10 $\mu 1$ amounts of a solution of $10 \mathrm{mg} / \mathrm{ml}$ ) was added to each well and, $4 \mathrm{~h}$ later, the solution was removed, and DMSO (100 $\mu \mathrm{l})$ added to each well. Percentages of absorbance relative to that of the control were plotted as linear functions of drug concentration. 
The $50 \%$ inhibitory concentration $\left(\mathrm{IC}_{50}\right)$ was the concentration of drug required to inhibit growth by $50 \%$, relative to that of the control. Cell growth inhibition was measured as the viable cell percentage relative to that of the control.

Transmission electron microscopy (TEM). CNE2 cells were seeded in $10 \mathrm{~cm}$-diameter dishes, cultured for $24 \mathrm{~h}$, and next treated with either DMSO [final concentration, $0.1 \%(\mathrm{v} / \mathrm{v})$ ] or ApoG2 in DMSO $(20 \mu \mathrm{M})$ for $24 \mathrm{~h}$. Cells were fixed in $4 \mathrm{ml}$ of ice-cold $2.5 \%(\mathrm{v} / \mathrm{v})$ glutaraldehyde of electron microscopy grade, rinsed with PBS, fixed in $1 \%$ (w/v) osmium tetroxide, dehydrated via passage through a graded series of ethanol baths $(50-90 \%, \mathrm{v} / \mathrm{v})$, washed in $90 \%(\mathrm{v} / \mathrm{v})$ acetone, and embedded in Epon 812 resin. Ultrathin (50-60 nm) sections were cut using an LKB NOVA ultramicrotome (LKB-NOVA, Bromma, Sweden). The sections were stained with $2 \%(\mathrm{w} / \mathrm{v})$ uranyl acetate and lead citrate, and visualized using a Philips CN10 transmission electron microscope at either x6,000 or x12,000 magnification.

Immunoblotting and immunoprecipitation. CNE1 and CNE2 cells were treated with $5 \mu \mathrm{M}$ ApoG2 for $0,6,12,24$ or $48 \mathrm{~h}$; or with $0,5,10$ or $20 \mu \mathrm{M}$ ApoG2 for $24 \mathrm{~h}$. Whole-cell lysates were prepared in $3 \mathrm{X}$ SDS sample buffer. Equal amounts of protein were electrophoresed on SDS-PAGE gels and transferred to polyvinylidene difluoride membranes. Membranes were next blocked for $60 \mathrm{~min}$ at room temperature with $5 \%(\mathrm{w} / \mathrm{v})$ nonfat dry milk/TBS-Tween-20, and reacted with antibodies detecting LC3, GAPDH, or p62, overnight at $4^{\circ} \mathrm{C}$ with gentle rocking. Membranes were next washed in TBS-Tween-20 and incubated with horseradish peroxidase-conjugated secondary antibodies for $1 \mathrm{~h}$ at room temperature. Proteins were visualized with the aid of reagents detecting enhanced chemiluminescence, followed by exposure to radiographic film.

When immunoprecipitation was planned, CNE2 cells were treated with $20 \mu \mathrm{M}$ ApoG2 for $24 \mathrm{~h}$, harvested, and lysed in ice-cold lysis buffer. Lysates were centrifuged at 12,000 x g for $15 \mathrm{~min}$, and the supernatants incubated overnight at $4^{\circ} \mathrm{C}$ with anti-Bcl-2 antibody. Antibody-protein complexes were pelleted with the aid of protein A-agarose. Precipitates were harvested by brief centrifugation and washed five times with ice-cold lysis buffer. Immunoprecipitated proteins were eluted with 3X SDS sample buffer and further analyzed by SDS-PAGE followed by immunoblotting with either anti-Bcl-2 or anti-Beclin 1 antibody to determine the effect of ApoG2 on the interaction of Beclin 1 and Bcl-2.

Plasmids and transfection. We used a retroviral construct (pSUPER puro, a gift of Professor Musheng Zeng, Cancer Center, Sun Yat-sen University, Guangzhou, China) encoding stable ATG5 siRNA with hairpin sequences. CNE2 cells stably expressing Atg5 shRNA were established by infection with retrovirus-containing supernatants, as described previously (33). After $24 \mathrm{~h}$ of transfection, cells were used in further experiments.

GFP-LC3 punctate staining assay. CNE2 cells were grown on glass coverslips and transfected with the pGFP-LC3 vector using Lipofectamine 2000 (Invitrogen Life Technologies, Carlsbad, CA, USA). Twenty-four hours later, the cells were divided into four groups: a control group, an ApoG2 group (20 $\mu \mathrm{M}$ ApoG2), an irradiation group (2 Gy irradiation), and an ApoG2-plus-irradiation group ( $20 \mu \mathrm{M}$ ApoG2 for $4 \mathrm{~h}$ and irradiation with $2 \mathrm{~Gy}$ ). After $24 \mathrm{~h}$, all cells were fixed in $4 \%$ (v/v) paraformaldehyde in PBS for $30 \mathrm{~min}$ at room temperature. Coverslips were mounted in anti-fade solution and stored at $4^{\circ} \mathrm{C}$ prior to examination using laser-scanning confocal microscopy (Olympus FV-1000).

Colony formation assay. CNE2 cells were irradiated with 0-6 Gy, as indicated, at a dose rate of $1.8 \mathrm{~Gy} / \mathrm{min}$, using a ${ }^{137}[\mathrm{Cs}]$ irradiator (Shepherd and Associates, Glendale, CA, USA). Irradiated cells were seeded in 12-well plates in RPMI-1640 with $10 \%$ (v/v) FBS, and incubated at $37^{\circ} \mathrm{C}$ for $12-14$ days. Cells were fixed for $15 \mathrm{~min}$ in $3: 1 \mathrm{(v/v)}$ methanol:acetic acid and stained for $15 \mathrm{~min}$ with $0.5 \%$ (w/v) crystal violet (Sigma, St. Louis, MO, USA) in methanol. After staining, colonies were counted using a cut off of 50 viable cells/colony. A 'surviving fraction' was calculated as (mean colony counts)/ (cells inoculated) $x$ [plating efficiency (PE)], where PE was defined as (mean colony counts)/(cells inoculated) for nonirradiated controls. Experiments were performed in triplicate and mean, SD and P-values calculated.

In vivo treatment and immunohistochemistry. Athymic nude (nu/nu) mice from the Animal Center of Guangzhou University of Traditional Chinese Medicine (Guangdong, China) received subcutaneous injections of $2 \times 10^{6} \mathrm{CNE} 2$ cells into each axilla. When the weights of subcutaneous tumors attained $>1,500 \mathrm{mg}$, mice were euthanized, and tumors dissected and mechanically dissociated into equally sized pieces prior to transplantation into the flanks of a new group of mice. When xenograft tumors became palpable $\left(\sim 0.1 \mathrm{~mm}^{3}\right.$ in volume), mice were randomly divided into a control group [receiving a $0.5 \%(\mathrm{w} / \mathrm{v})$ sodium carboxymethylcellulose solution]; an ApoG2 group (120 mg ApoG2 per kg of body weight intragastrically daily for 7 continuous days); a 2 Gy group (irradiation with 2 Gy of ${ }^{60}[\mathrm{Co}] \gamma$-rays divided into five equal fractions given over 5 consecutive days); and an ApoG2-plus-2 Gy combination group. Each group had four mice, and tumor size did not differ among groups. Tumor volumes were calculated every 3 days, using the formula $\mathrm{V}=\mathrm{ab}^{2} \pi / 6$, where a was the greatest tumor diameter and $b$ the shortest. After treatment for 20 days, mice were euthanized and tumors were dissected and weighed. Immunohistochemical analysis was performed on sections of CNE2 xenografts from the four groups. All samples were stained with hematoxylin and eosin and microscopically examined to confirm that the tumors originated from CNE2 cells. Sections were next incubated with anti-LC3 antibody at $4^{\circ} \mathrm{C}$ overnight and visualized using diaminobenzidine (DAB) as a peroxidase substrate. The experiment protocol was approved by Sun Yat-sen University Cancer Center ethics committee for animal experiment.

Statistical analysis. The significance of between-group differences were compared using the unpaired t-test of SPSS version 15.0 software.

\section{Results}

ApoG2 inhibits the proliferation of NPC cells. The chemical structures of gossypol and the optimized derivative 


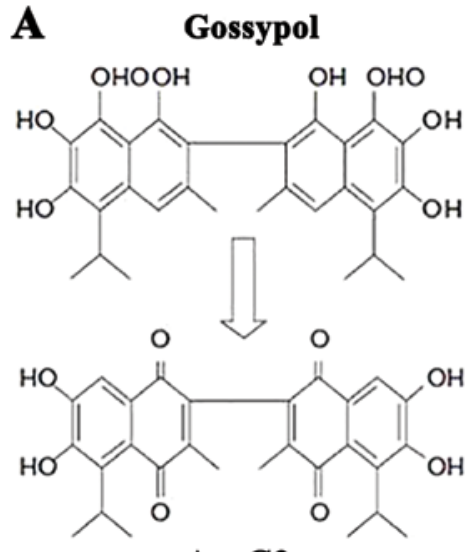

ApoG2

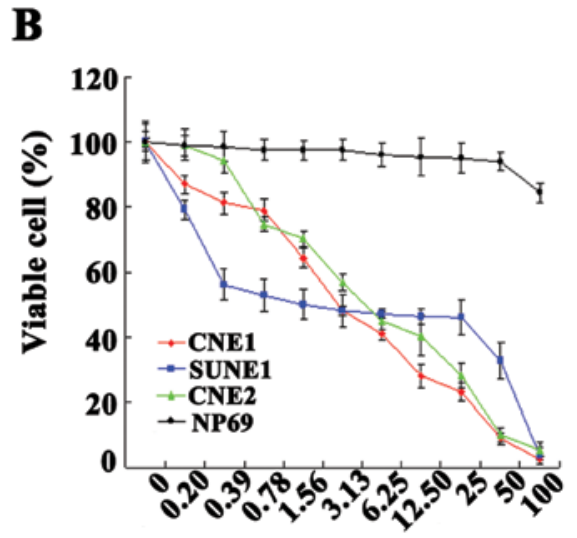

Concentration $(\boldsymbol{\mu M})$

Figure 1. ApoG2 and the inhibitory effect on proliferation of NPC cells. (A) The chemical structures of gossypol and the derivative apogossypolone (ApoG2). (B) ApoG2 inhibited the proliferation of CNE1, CNE2, SUNE1 and NP69 cells. CNE1 (red line), CNE2 (green line), SUNE1 (blue line) and NP69 (black line) cells were treated with the indicated concentrations of ApoG2 for $72 \mathrm{~h}$ and cell viability was determined by the MTT assay, as described in Materials and methods.
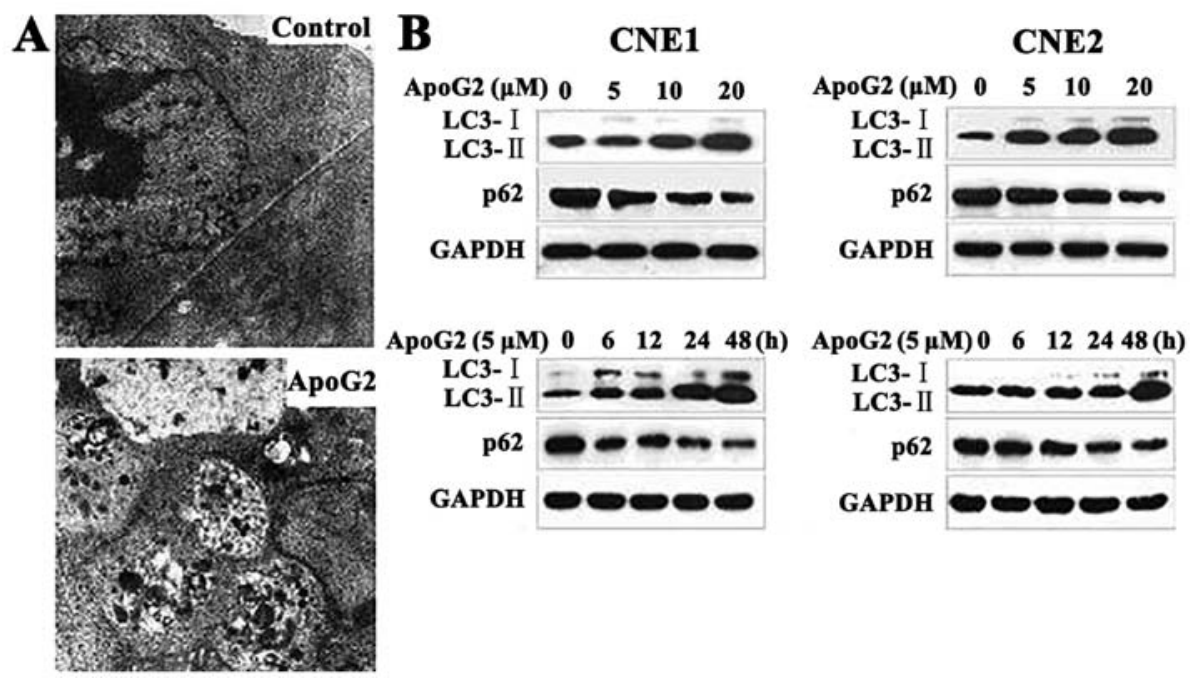

Figure 2. ApoG2 induced autophagy of NPCs. (A) ApoG2 induced autophagy of CNE2 cells. Representative transmission electron photomicrographs of CNE2 cells treated with $20 \mu \mathrm{M} \mathrm{ApoG} 2$ for $24 \mathrm{~h}$. Both the nuclear and general morphologies of control cells were normal (top image, x6,000). Exposure to ApoG2 for $24 \mathrm{~h}$ resulted in development of autophagic vacuoles (bottom image, $\mathrm{x} 12,000$ ) containing remnants of organelles. (B) ApoG2 concentration- and time-dependently induced expression of LC3-II and degradation of p62; both are autophagic markers. CNE1 and CNE2 cells were treated with ApoG2 at the indicated concentrations for $24 \mathrm{~h}$, or $5 \mu \mathrm{M}$ ApoG2 for the indicated times. Lysates were analyzed via immunoblotting using anti-LC3 and p62 antibodies.

ApoG2 (34), an oxidation product of gossypol, lacking two aldehyde groups are shown in Fig. 1A. The NPC cell lines CNE1, CNE2 and SUNE1, and the immortalized NP69 cell line, were exposed to different concentrations of ApoG2 for 72 h. As shown in Fig. 1B, ApoG2 dose-dependently inhibited the viability of CNE1, CNE2 and SUNE1 cells, but not that of NP69 cells. At $100 \mu \mathrm{M}$, ApoG2 reduced proliferation of CNE1, CNE2 and SUNE1 cells by $~ 90 \%$ over $72 \mathrm{~h}$. In contrast, when NP69 cells were exposed to different concentrations of ApoG2, no obvious inhibition was observed even at $\leq 50 \mu \mathrm{M}$ ApoG2 (Fig. 1B). At $72 \mathrm{~h}$, the $\mathrm{IC}_{50}$ values of ApoG2 acting on CNE1, CNE2, and SUNE1 cells were $2.84 \pm 0.19,2.18 \pm 0.38$ and $5.64 \pm 0.65 \mu \mathrm{M}$, respectively.

ApoG2 induces autophagy of NPC cells. Autophagy is an evolutionarily conserved process of protein degradation associated with tumor promotion and tumor suppression in different situations. To determine whether ApoG2 could induce autophagy of NPC cells, we monitored morphological changes in such cells via transmission electron microscopy. As shown in Fig. 2A, no autophagic vacuoles were observed in DMSO-treated CNE2 cells. CNE2 cells incubated with $20 \mu \mathrm{M}$ ApoG2 for $24 \mathrm{~h}$ developed large cytoplasmic vacuoles with membrane bilayers. The vacuoles resembled autophagosomes and contained remnants of degraded organelles.

These results were confirmed by western blotting. The microtubule-associated protein 1 light chain 3 (LC3), a homolog of yeast Atg8, is present on isolated autophagosomal membranes. The amount of LC3-II correlates well with the number of autophagosomes. Enhancement of conversion of LC3-I to LC3-II, and upregulation of LC3 expression, occurs when autophagy is induced (35). Protein p62 (also known as 


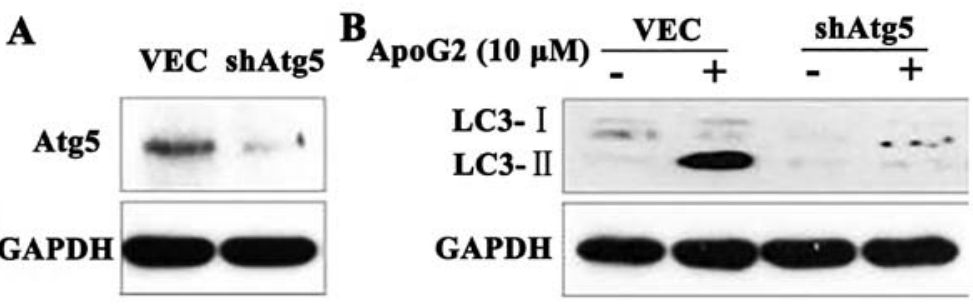

Figure 3. Atg5 is required for ApoG2-induced autophagy. (A) The efficiency of shRNA Atg5 transfection. (B) shAtg5 blocked autophagy induced by ApoG2. Immunoblotting showed that LC3-II was expressed in CNE2/vector cells after treatment with $10 \mu \mathrm{M}$ ApoG2 for $24 \mathrm{~h}$, and it was not expressed in CNE2/shAtg5 cells after the same treatment.

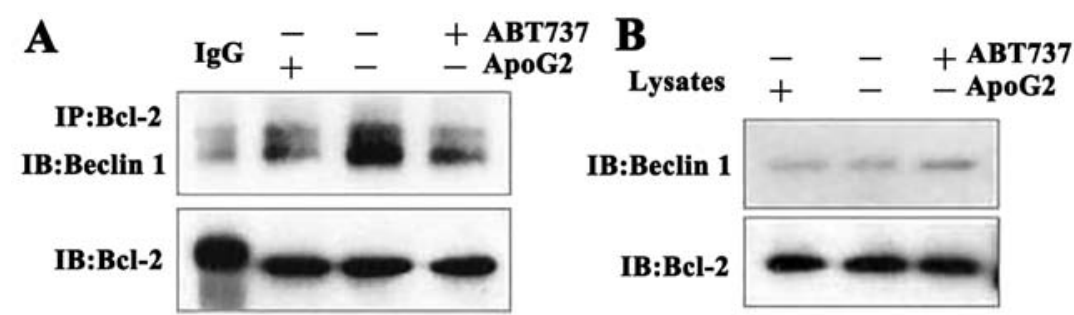

Figure 4. Inhibition by ApoG2 of the binding of Bcl-2 to Beclin 1 in CNE2 cells. CNE2 cells $\left(1 \times 10^{6}\right)$ were treated with $10 \mu \mathrm{M}$ of ApoG 2 or $20 \mu \mathrm{M}$ ABT-737 for $24 \mathrm{~h}$. Immunoprecipitation was performed as described in Materials and methods. Equivalent amounts of cell lysates were immunoprecipitated with primary antibodies to Bcl-2 [or (control) normal rabbit IgG]. Western blotting was performed to explore whether ApoG2 treatment affected binding of Beclin 1 to Bcl-2. ABT737 was used as a positive control $(20,21)$.

SQSTM1) has a short region that interacts with LC3.p62 participates in autophagy and is degraded in autolysosomes (36). Conversion of LC3-I to LC3-II and p62 degradation are two reliable markers of autophagy. After CNE1 and CNE2 cells were treated with ApoG2 at different concentrations or for different times, LC3 and p62 expression levels were measured by immunoblotting. Expression of LC3-II increased whereas that of p62 protein decreased in CNE1 and CNE2 cell lines treated with ApoG2, in a dose- and time-dependent manner (Fig. 2B).

Atg5 is required for ApoG2-induced autophagy. Atg5 is required for formation of autophagosomes, and Atg5-deficient mouse embryonic stem cells exhibited significantly reduced numbers of autophagic vesicles (37). To confirm the role played by autophagy in this process, we used CNE2-shAtg5 cells. We found that CNE2-shAtg5 cells lacked Atg5 expression (Fig. 3A). When CNE2 cells treated with $10 \mu \mathrm{M}$ ApoG2 were additionally transfected with a plasmid expressing shAtg5, or not, expression of LC3-II in CNE2-shAtg5 cells decreased over $24 \mathrm{~h}$, compared with control cells (Fig. 3B). This indicated that Atg5 was required for ApoG2-induced autophagy.

ApoG2 blocks binding of Bcl-2 to Beclin 1. We earlier reported that ApoG2 blocked the anti-apoptotic functions of Bcl-2 family proteins without affecting the expression levels of these proteins, rather inhibiting the binding of Bcl-2 and Bcl-xL to Bax in CNE2 cells (13). and that of Bcl-2 to Bax in U937 cells (30). The BH3 mimetic ABT-737 competitively dissociated Beclin 1 from the pro-survival Bcl-2/Bcl-xL complex, thereby inducing autophagy (38). Gossypol, another mimic of the Bcl-2 homology domain 3 induced autophagy in both MCF-7 and HeLa cells, and inhibited the binding of Bcl-2 to Beclin 1 in MCF-7 cells, but not HeLa cells. ABT-737 inhibited the binding of Bcl-2 to Beclin1 in HeLa cells (39). We speculated that ApoG2 might trigger autophagy of CNE2 cells by influencing the interaction of an anti-apoptotic protein (Bcl-2) with a pro-autophagic and pro-apoptotic protein (Beclin 1). To verify this hypothesis, whole lysates from treated and untreated CNE2 cells were collected, immunoprecipitated with an anti-Bcl-2 specific antibody, and next probed with an anti-Beclin 1 specific antibody. As shown in Fig. 4, Bcl-2 and Beclin 1 bound to each other in untreated CNE2 cells. However, when cells were exposed to $10 \mu \mathrm{M}$ of ApoG2 or $20 \mu \mathrm{M}$ ABT-737 for $24 \mathrm{~h}$, such binding was eliminated. These results showed that ApoG2 inhibited heterodimerization of Bcl-2 and Beclin 1 in CNE2 cells.

ApoG2-mediated autophagy contributes to the radiation sensitivity of NPC cells. We used the colony-forming test to demonstrate the radiosensitizing effect of ApoG2. CNE2 cells were treated with ApoG2 in combination with ionizing radiation. Surviving colonies were counted 14 days later. Survival decreased, in a dose-dependent manner, when cells were treated with $0.5,1$ and $2 \mu \mathrm{M}$ of ApoG2 for 14 days. All DEFs (dose enhancement factors) were greater than unity. The DEFs of radiation-plus-continuous ApoG 2 at 2,1 or $0.5 \mu \mathrm{M}$ were $1.92,1.12$ and 1.12, respectively. The SFs (survival fractions) were $0.81,0.83$ and 0.86, respectively. Thus, ApoG2 enhanced radiosensitization of $\mathrm{CNE} 2$ cells in a dose-dependent manner (Fig. 5A and B).

Autophagy is an evolutionarily conserved process of protein degradation associated with both tumor promotion and suppression in different situations. Autophagy contributes to cell killing following radiation. We explored whether ApoG2 


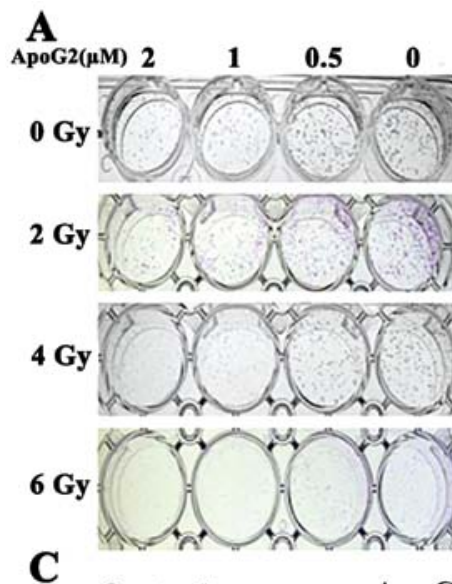

B
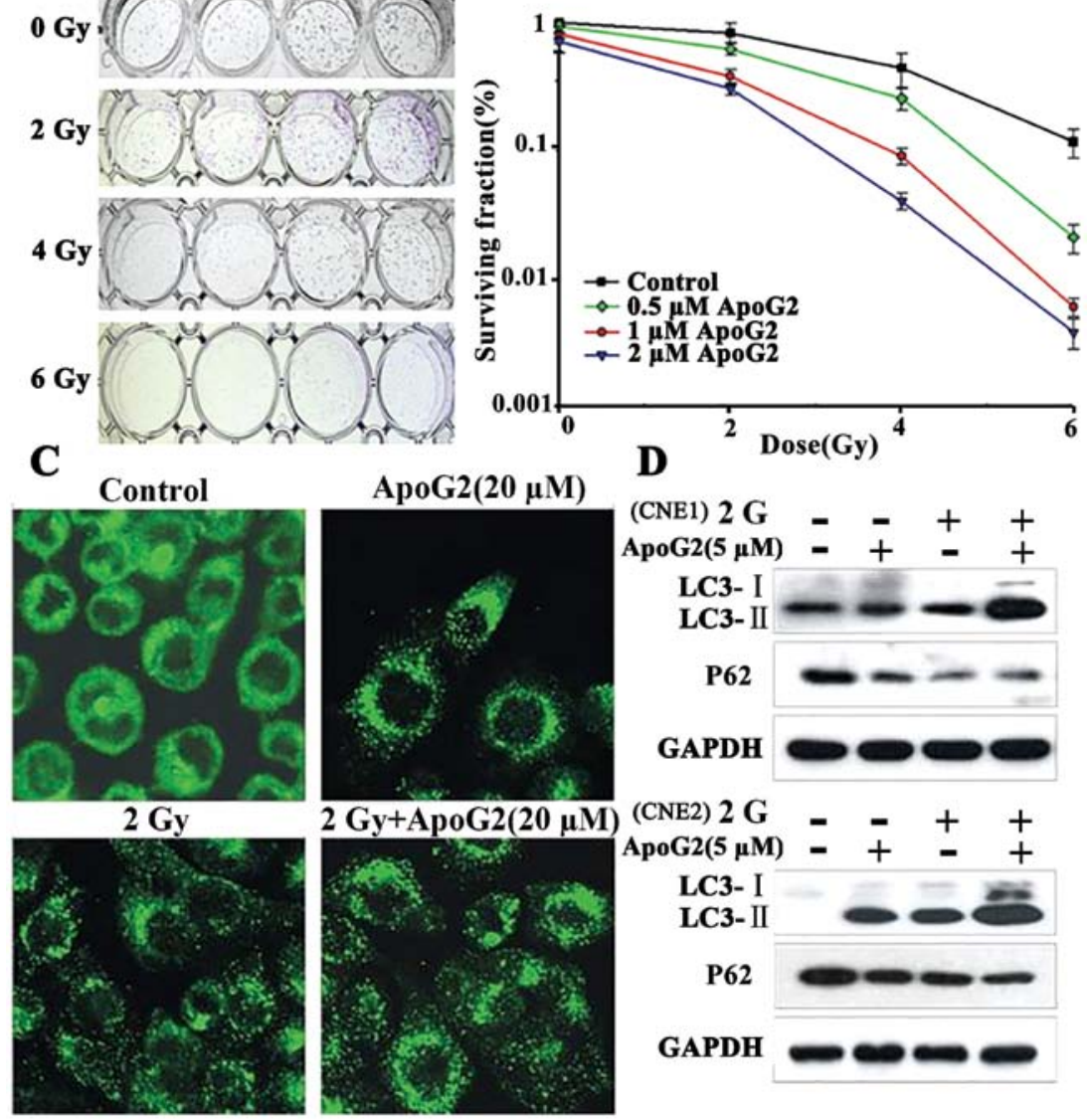

Figure 5. ApoG2 enhanced radiation-mediated autophagy and apoptosis of NPC cells. (A) Colony-forming test results. CNE2 cells were incubated with ApoG2 $(0,0.5,1$, or $2 \mu \mathrm{M})$ with or without radiation $(0,2,4$, or $6 \mathrm{~Gy})$. Cells were irradiated to various extents and seeded into 12 -well plates with different concentrations of ApoG2. After 14 days of incubation, cells were stained with crystal violet, and the numbers of colonies containing $>50$ cells were counted. Values are the means \pm SD of data from triplicate experiments. (B) SFs (survival fractions) were calculated. (C) Expression and localization of GFP-LC3 in CNE2 cells treated with ApoG2, 2 Gy, or the combination, for 24 h. (D) Autophagy was induced by ApoG2 combined with radiation. CNE1 and CNE2 cells were treated (or not) with $5 \mu \mathrm{M}$ of ApoG2 for $4 \mathrm{~h}$ and next irradiated with $2 \mathrm{~Gy}$. After $24 \mathrm{~h}$, cells were subjected to immunoblotting using anti-LC3 and -p62 antibodies.

could enhance radiosensitivity by stimulating autophagy. First, CNE1 and CNE2 cells were transfected with a plasmid expressing LC3 fused to green fluorescent protein (GFP-LC3). Cells exhibiting punctate GFP signaling were considered to be autophagic because the reporter protein was lysosomally located, as is characteristic of LC3 during autophagy. In control cells, GFP-LC3 was evenly distributed throughout the entire cytoplasm. After cells were exposed to $20 \mu \mathrm{M}$ ApoG2, ring-shaped structures were easily detectable in the cytosol, indicating that GFP-LC3 protein associated with autophagosomal membranes, demonstrating induction of autophagy. After treatment with ApoG2 and radiation, more ring-shaped structures were evident in the cytosol than after treatment with ApoG2 alone (Fig. 5C).

These results were confirmed by measurement of the levels of processed LC3 and p62. After treatment with ApoG2 and radiation, LC3 and p62 expression levels were measured via immunoblotting. LC3-II expression in both CNE1 and CNE2 cell lines treated with ApoG2 $(5 \mu \mathrm{M})$ and radiation (2 Gy) increased compared with the control, ApoG2-alone $(5 \mu \mathrm{M})$, and radiation-alone (2 Gy) groups, and the p62 expression level fell (Fig. 5D).
ApoG2 enhances the radiosensitivity of CNE2 xenografts in nude mice. The most prominent antitumor effect was observed when ApoG2 and radiation were given in combination. ApoG2 was well tolerated by the nude mice. The maximal tolerated dose (MTD) was previously found to be over $480 \mathrm{mg} / \mathrm{kg}$, upon daily intragastric administration. Fig. 6A shows the tumor volumes of mice given ApoG2 (120 mg/kg intragastrically daily for 7 days), mice irradiated with 2 Gy (using 60[Co] $\gamma$-rays for 5 continuous days), and the ApoG2-plus-2 Gy combination group, compared with the control group. The tumor volumes of the combination group decreased significantly compared those of the other two test groups $(\mathrm{P}<0.05)$. When it was estimated that all tumors in the control group had grown to weigh over $1,000 \mathrm{mg}$, the mice were sacrificed and xenografts removed for weighing (Fig. 6B). Antitumor activity (inhibitory rate) measurements for the ApoG2, $2 \mathrm{~Gy}$, and combination groups [measured by calculation of (C-T)/C percentage ratios] were 46.89, 19.34, and $61.64 \%$, respectively (Table I).

To determine whether ApoG2 or radiation induced autophagy of NPC cells in vivo, tumor sections from CNE2 xenografts were incubated with anti-LC3 antibody. When the 
A

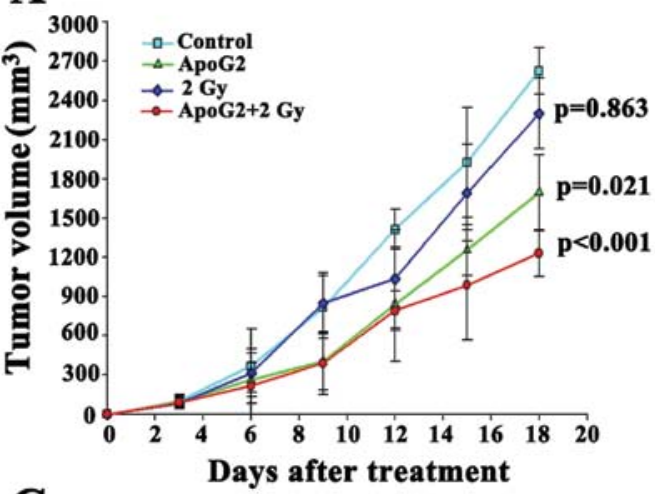

C

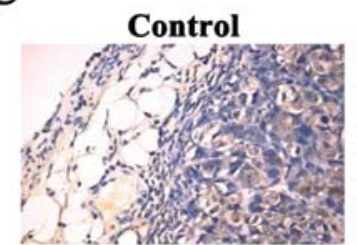

5*2 Gy

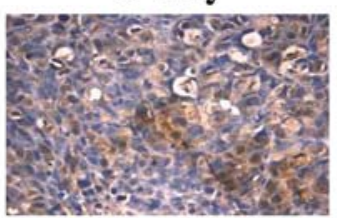

B

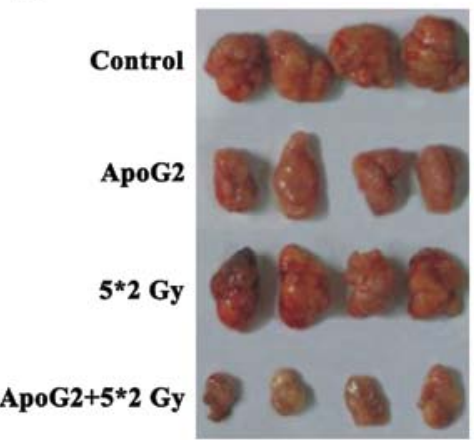

D

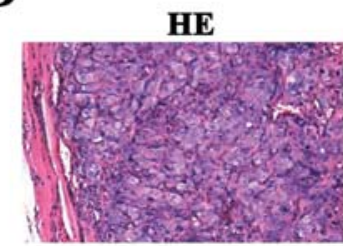

Negative

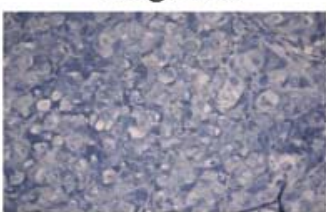

Figure 6. ApoG2 enhanced the radiosensitivity of CNE2 xenografts in nude mice. We measured the inhibition of tumor growth by ApoG2, 2 Gy of radiation, or the combination, in CNE2 xenograft-bearing nude mice. Maximal and minimal tumor diameters (the a and $b$ values, respectively) were measured every 3 days (A). Tumor volumes were calculated as $v=a b 2 \pi / 6$. Control and test data were compared. After 18 days, the experiment was terminated. (B) The tumor growth inhibition ratios [100x(1-T/C) (\% values)] were calculated by dividing the average tumor volume of the treatment group by that of the control group. $\left({ }^{*} \mathrm{P}<0.05,{ }^{* *} \mathrm{P}<0.01\right.$; Table I). Tumor tissues from each group (control, ApoG2, 2 Gy and the ApoG2-plus-2 Gy combination) were obtained at the end of treatment (C). Immunohistochemical staining for LC3 of CNE2 xenograft tumor sections after treatment. (D) H\&E staining, and staining of the negative control. Magnification, $\mathrm{x} 400$.

Table I. Tumor growth inhibition ratios (\%).

\begin{tabular}{lccc}
\hline Group & $\mathrm{n}$ & $\begin{array}{c}\text { Weight of } \\
\text { tumor }(\mathrm{g})\end{array}$ & $\begin{array}{c}\text { Inhibitory } \\
\text { rate }(\%)\end{array}$ \\
\hline Control & 4 & $3.05 \pm 0.63$ & - \\
ApoG2 & 4 & $1.62 \pm 0.89$ & $46.89 \pm 5.57^{\mathrm{a}}$ \\
$2 \mathrm{~Gy}$ & 4 & $2.64 \pm 0.43$ & $19.43 \pm 1.82$ \\
ApoG2+2 Gy & 4 & $1.17 \pm 0.87$ & $61.64 \pm 5.79^{\mathrm{b}}$ \\
\hline
\end{tabular}

${ }^{\mathrm{a}} \mathrm{P}<0.05,{ }^{\mathrm{b}} \mathrm{P}<0.01$

weights of control xenografts exceeded $1,000 \mathrm{mg}$, all mice were euthanized and tumors were dissected, weighed, and fixed for immunochemical examination. As shown in Fig. 6C, treatment with a combination of ApoG2 and 2 Gy of radiation stimulated a significant rise in LC3 expression in CNE2 xenografts compared to controls.

\section{Discussion}

Nasopharyngeal carcinoma (NPC) is the most common malignancy in China. The annual morbidity rate is 10-25/100,000. Of all Chinese NPC patients, 60\% live in
Guangdong province. The primary treatment for NPC is radiotherapy. The 5-year survival rate of early-stage NPC patients treated with radiotherapy is $80-90 \%$. In patients with recurrent NPC who receive a second course of radiation therapy, and who experience severe radiation-induced damage, the 5-year survival rate drops to $12-40 \%$. Further, a third course of radiation is even less effective (4). Therefore, it is very important to explore new ways to enhance the sensitivity of NPC cells to radiation.

Autophagy is one response of cancer cells to various therapies, including ionizing radiation. Radiation induces autophagy, but not apoptosis, of various malignant tumor cell lines. Beclin 1, an essential autophagic protein, was recently identified as a BH3 domain-only protein that binds to members of the Bcl-2 anti-apoptotic family (40). Dissociation of Beclin 1 from Bcl-2 inhibitors is essential if the former protein is to exhibit autophagic activity. Apogossypolone (ApoG2) is a novel derivative of gossypol, a small-molecule inhibitor of anti-apoptotic Bcl-2 family proteins (39). We hypothesized that ApoG2 was both a Bcl-2 inhibitor and exerted a radiosensitizing effect by activating autophagy.

In the current study, we used human NPC cell lines (CNE1, CNE2, and SUNE1), and a normal human nasopharyngeal epithelial cell line (NP69), to study the inhibitory effect of ApoG2. We incubated cells with ApoG2 (at different concentrations) for $72 \mathrm{~h}$. Remarkable inhibitory effects were 
observed. The $\mathrm{IC}_{50}$ values for CNE1, CNE2 and SUNE1 cells were $2.84,2.18$ and $5.64 \mu \mathrm{M}$, respectively. However, ApoG2 had no obvious effect on normal NP69 cells.

The adaptor protein p62, also termed sequestosome 1 (SQSTM1), binds to both LC3 and to ubiquitinated proteins to facilitate autophagic clearance. p62 accumulates when autophagy is inhibited, and falls when autophagy is induced. Therefore, p62 can be used as a marker of autophagic flux (41). LC3 is an autophagosomal ortholog of yeast Atg8. A lipidated form of LC3, LC3-II, has been shown to serve as an autophagosomal marker in mammals (35). We earlier found that ApoG2 induced apoptosis of human lymphoma U937 and NPC cells (30). In the present study, we found that expression of LC3-II increased and that of p62 decreased, in a doseand time-dependent manner, when CNE1 and CNE2 cells were treated with ApoG2. This means that ApoG2 induced autophagy in these two cell types (Fig. 2). Autophagosomes were evident in transmission electron micrographs of CNE2 cells treated with ApoG2 for $24 \mathrm{~h}$ (Fig. 2). When CNE2 cells were transfected with the GFP-LC3 plasmid, ring-shaped structures were detectable in the cytosol of the ApoG2 group, indicating that autophagy was in play (Fig. 2). Thus, three different experimental approaches showed that ApoG2 induced autophagy of NPC cells.

To further confirm that ApoG2 induced autophagic death of NPC cells, CNE2 cells were treated with shRNA targeting Atg5 expression. The Atg5 protein is required for formation of autophagosomes and plays an important role in vesicle expansion and completion (42). In the present study, western blotting indicated that shRNA targeting Atg5 significantly reduced expression of both Atg 5 as well as LC3-II in cells treated with ApoG2. This means that ApoG2 induced autophagy of CNE2 cells and that Atg 5 was involved in this process.

How does ApoG2 induce autophagy? It is known that Bcl-2 and close homologs thereof, including Bcl-xL and Mcl-1, interact with the evolutionarily conserved autophagic protein Beclin 1, and inhibit Beclin 1-dependent autophagy in yeast and mammalian cells. BH3-only proteins stimulate autophagy by competitively disrupting the interaction between Beclin 1 and $\mathrm{Bcl}-2 / \mathrm{Bcl}-\mathrm{xL}$, thus releasing Beclin 1 from its inhibition (40). ABT-737 disrupts the interaction between Beclin 1 and Bcl-2 in HeLa cells, thereby liberating Beclin 1 from an inhibitory complex $(38,39)$. Lian et al found that a small-molecule inhibitor of Bcl-2, (-)-gossypol, abolished $\mathrm{Bcl}-2 / \mathrm{Beclin} 1$ interaction in prostate $\mathrm{CL}-1$ cancer cells (43). Gao et al used MCF-7 and HeLa cells, which express detectable endogenous levels of both Beclin 1 and $\mathrm{Bcl}-2$, to investigate the effect of gossypol on dissociation of these proteins (39). Co-immunoprecipitation experiments showed that when gossypol induced autophagy, dissociation of the Beclin 1/Bcl-2 complex was observed in MCF-7 cells, but not HeLa cells, as was also the case when cells were starved. In a previous study, we found that ApoG2, the oxidation product of apogossypol, blocked formation of the $\mathrm{Bcl}-2 / \mathrm{Bax}$ (Bcl-xL/Bak) complex, triggering activation of the mitochondrial apoptotic pathway in NPC CNE2 (13) and human lymphoma U937 cells (30). Thus, we hypothesized that ApoG2 might inhibit the binding of Bcl-2 to Beclin 1.
We used ABT-737 as a positive control, and found that when CNE2 cells were exposed to $10 \mu \mathrm{M}$ ApoG2 or $20 \mu \mathrm{M}$ ABT-737 for $24 \mathrm{~h}$, the binding of Bcl-2 to Beclin 1 was inhibited. Thus, ApoG2 inhibited the heterodimerization of Bcl-2 with Beclin 1 in CNE2 cells, triggering release of the BH3-only pro-autophagic protein Beclin 1, in turn triggering the autophagic cascade.

NPC is highly radiosensitive. Thus, radiotherapy (RT) plays a central role in the treatment of all stages of NPC that lack distant metastases. As RT achieves good local control, distant metastatic failure has become the most common pattern of recurrence, especially among those with locoregionally advanced disease, which is difficult to control using conventional 2D RT. It is thus important to improve NPC radiosensitivity. The rationale of induction chemotherapy is to reduce the tumor load of locoregional disease prior to RT commencement, and to prescribe early systemic treatment for eradication of micro-metastases. The International Nasopharyngeal Carcinoma Study Group reported that a combination of bleomycin, epirubicin, and cisplatin significantly improved disease-free, but not overall, survival. This may be attributable to elevated numbers of treatment-related deaths among patients on induction chemotherapy compared to RT alone (8\% vs. $1 \%$ ) (4).

Radiotherapy induced apoptosis and autophagy of NPC cells but the cells continued to express the $\mathrm{Bcl}-2$ protein at a high level, Bcl-2 mediates resistance to apoptosis and autophagy (44). Thus, we sought a new approach toward inhibition of Bcl-2 function, to improve the radiosensitivity of NPC cells. Earlier, we showed that ApoG2 induced apoptosis in NPC cells by blocking the binding of Bcl-2 to Bax. In the present study, we found that ApoG2 induced autophagy of NPC cells.

We found that ApoG2 could radiosensitize the human NPC CNE2 cell line in vitro. The clone-forming assay revealed that the DEFs of radiation given in the continuous presence of 2,1 or $0.5 \mu \mathrm{M}$ ApoG2 were 1.92, 1.12 and 1.12 , respectively. The SFs of groups given radiation in the continuous presence of $2 \mu \mathrm{M}$ ApoG2 was 0.81 , and those of groups given 1 and $0.5 \mu \mathrm{M}$ of ApoG2 0.83 and 0.86 . These results indicated that ApoG2 enhanced radiosensitization of CNE2 cells in a time-dependent manner. We next tested the extent of autophagy of CNE1 and CNE2 cells by western blotting and confocal microscopy. ApoG2-plus-radiation induced more autophagy, increased punctate GFP signaling, raised the expression level of LC3-II, and reduced that of P62, compared to the ApoG- or radiation-alone groups, and controls.

Next, we explored the anticancer effects of ApoG2 in nude mice, We found that ApoG2 combined with radiation suppressed the growth of CNE2 tumor xenografts in nude mice more effectively that did ApoG2 or radiation alone. ApoG2 radiosensitized NPC cells in vivo as revealed by the (C-T)/C ratios (in percentages). The images for the ApoG2, radiation, and combination groups were 46.89, 19.34, and $61.64 \%$, respectively (Fig. 5C). Immunohistological staining showed that LC3-II levels became gradually upregulated in the ApoG2-plus-radiation group (Fig. 5D).

In conclusion, we have shown that the BH3-mimetic ApoG2 induced autophagic death of human NPC cells. 
ApoG2 radiosensitized CNE1 and CNE2 cells via induction of autophagy, triggered by inhibition of the binding of Bcl-2 to Beclin 1. This is the potential mechanism by which ApoG2 acts in NPC cells. ApoG2 exhibited anticancer and radiosensitizing effects on CNE2 xenografts in nude mice. These findings suggest that autophagy is one mechanism triggered by ApoG2 and that enhancement of autophagy can be used to complement combination therapy with ionizing radiation when NPC is to be treated.

\section{Acknowledgements}

This study was supported by the National Nature Science Foundation of China (30873085), 973 Program (2011CB504300), the Nature Science Foundation of Guangdong (10451008901004533) and the technical New Star of Zhujiang, Pan Yu districts, Guangzhou (2013-special15-6.09).

\section{References}

1. McDermott AL, Dutt SN and Watkinson JC: The aetiology of nasopharyngeal carcinoma. Clin Otolaryngol Allied Sci 26 82-92, 2001

2. Bei JX, Jia WH and Zeng YX: Familial and large-scale casecontrol studies identify genes associated with nasopharyngeal carcinoma. Semin Cancer Biol 22: 96-106, 2012.

3. Lee AW, Lin JC and Ng WT: Current management of nasopharyngeal cancer. Semin Radiat Oncol 22: 233-244, 2012.

4. Yoshizaki T, Ito M, Murono S, Wakisaka N, Kondo S and Endo K: Current understanding and management of nasopharyngeal carcinoma. Auris Nasus Larynx 39: 137-144, 2012.

5. Kang $\mathrm{MH}$ and Reynolds $\mathrm{CP}$ : Bcl-2 inhibitors: targeting mitochondrial apoptotic pathways in cancer therapy. Clin Cancer Res 15: 1126-1132, 2009.

6. Zhou FF, Yang Y and Xing D: Bcl-2 and Bcl-xL play important roles in the crosstalk between autophagy and apoptosis. FEBS J 278: 403-413, 2011.

7. Levine B, Sinha S and Kroemer G: Bcl-2 family members: dual regulators of apoptosis and autophagy. Autophagy 4: 600-606, 2008

8. Green DR and Evan GI: A matter of life and death. Cancer Cell 1: 19-30, 2002.

9. Lu QL, Elia G, Lucas S and Thomas JA: Bcl-2 proto-oncogene expression in Epstein-Barr-virus-associated nasopharyngeal carcinoma. Int J Cancer 53: 29-35, 1993.

10. Fan SQ, Ma J, Zhou J, Xiong W, Xiao BY, Zhang WL, Tan C Li XL, Shen SR, Zhou M, Zhang QH and Ou YJ: Differential expression of Epstein-Barr virus-encoded RNA and several tumor-related genes in various types of nasopharyngeal epithelial lesions and nasopharyngeal carcinoma using tissue microarray analysis. Hum Pathol 37: 593-605, 2006.

11. Sheu, LF, Chen A, Meng CL, Ho KC, Lin FG and Lee WH: Analysis of bcl-2 expression in normal, inflamed, dysplastic nasopharyngeal epithelia, and nasopharyngeal carcinoma: association with p53 expression. Hum Pathol 28: 556-562, 1997.

12. Yu Y, Dong W, Li X, Yu E,Zhou X and Li S: Significance of c-Myc and Bcl-2 protein expression in nasopharyngeal carcinoma. Arch Otolaryngol Head Neck Surg 129: 1322-1326, 2003.

13. Hu ZY, Zhu XF, Zhong ZD, Sun J, Wang J, Yang D and Zeng YX: ApoG2, a novel inhibitor of antiapoptotic Bcl-2 family proteins, induces apoptosis and suppresses tumor growth in nasopharyngeal carcinoma xenografts. Int J Cancer 123: 2418-2429, 2008.

14. Del Poeta G, Venditti A, Del Principe MI, et al: Amount of spontaneous apoptosis detected by $\mathrm{Bax} / \mathrm{Bcl}-2$ ratio predicts outcome in acute myeloid leukemia (AML). Blood 101: 2125-2131, 2003.

15. Minn AJ, Rudin CM, Boise LH and Thompson CB: Expression of bcl-xL can confer a multidrug resistance phenotype. Blood 86: 1903-1910, 1995
16. Yoshino T, Shiina H, Urakami S, et al: Bcl-2 expression as a predictive marker of hormone-refractory prostate cancer treated with taxane-based chemotherapy. Clin Cancer Res 12: 6116-6124, 2006.

17. Lacy J, Loomis R, Grill S, Srimatkandada P, Carbone R and Cheng YC: Systemic Bcl-2 antisense oligodeoxynucleotide in combination with cisplatin cures EBV1 nasopharyngeal carcinoma xenografts in SCID mice. Int J Cancer 119: 309-316, 2006.

18. Oltersdorf T, Elmore SW, Shoemaker AR, Armstrong RC, Augeri DJ, Belli BA, Bruncko M, Deckwerth TL, Dinges J, Hajduk PJ, Joseph MK and Kitada S: An inhibitor of Bcl-2 family proteins induces regression of solid tumours. Nature 435: $677-681,2005$

19. Kang MH, WanZ,Kang Y,Sposto R and Reynolds CP: Mechanism of synergy of N-(4-hydroxyphenyl) retinamide and ABT-737 in acute lymphoblastic leukemia cell lines: Mcl-1inactivation. J Natl Cancer Inst 100: 580-595, 2008.

20. Konopleva M, Contractor R, Tsao T, et al: Mechanisms of apoptosis sensitivity and resistance to the $\mathrm{BH} 3$ mimetic ABT-737 in acute myeloid leukemia. Cancer Cell 10: 375-388, 2006.

21. Chauhan D, Velankar M, Brahmandam M, et al: A novel Bcl-2/ $\mathrm{Bcl}-\mathrm{X}(\mathrm{L}) / \mathrm{Bcl}-\mathrm{w}$ inhibitor ABT-737 as therapy in multiple myeloma. Oncogene 26: 2374-2380, 2006.

22. Van Delft MF, Wei AH, Mason KD, et al: The BH3 efficiently induces apoptosis via Bak/Bax if Mcl-1 is neutralized. Cancer Cell 10: 389-399, 2006.

23. Del GaizoMooreV, Brown JR, Certo M, Love TM, Novina CD and Letai A: Chronic lymphocytic leukemia requires BCL2 to sequester prodeath BIM, explaining sensitivity to BCL2 antagonist ABT-737. J Clin Invest 117: 112-121, 2007.

24. Lin X, Morgan-Lappe S and Huang X: 'Seed' analysis of offtarget siRNAs reveals an essential role of Mcl-1 in resistance to the small-molecule Bcl-2/Bcl-X(L) inhibitorABT-737. Oncogene 26: 3972-3979, 2007

25. Hann CL, Daniel VC, Sugar EA, Dobromilskaya I, Murphy SC, Cope L, Lin X, Hierman JS, Wilburn DL, Watkins DN and Rudin CM: Therapeutic efficacy of ABT-737, a selective inhibitor of BCL-2, in small cell lung cancer. Cancer Res 68: 2321-2328, 2008.

26. MacVicar GR, Kuzel TM and Curti BD: An open label, multicenter, phase I/II study of AT-101 in combination with docetaxel (D) and prednisone $(\mathrm{P})$ in men with hormone refractory prostate cancer (HRPC). J Clin Oncol 26: 16048, 2008.

27. Yang D, Chen J, Xu L, Gao W, Guo J, Qiu S, Holmlund J, Sorensen M and Wang S: AT-101 and ApoG2, highly potent and orally active small molecule inhibitors of Mcl-1 protein and potential application for apoptosis-targeted anticancer therapy. AACR-NCI-EORCT International Conference on Molecular Targets and Cancer Therapeutics. p255, abs. 223, Nov 14-18, 2005. http://www.aacr.org/Uploads/DocumentRepository/ pdf_files/2005MTCT/MT05_abstracts_C.pdf.

28. Arnold AA, Aboukameel A, Chen J, Yang D and Wang S: Preclinical studies of Apogossypolone: a new nonpeptidic pan small-molecule inhibitor of Bcl-2, Bcl-XL and Mcl-1 proteins in Follicular Small Cleaved Cell Lymphoma model. Mol Cancer 7: 20, 2008.

29. Dao VT, Dowd MK, Martin MT, Gaspard C, Mayer M and Michelot RJ: Cytotoxicity of enantiomers of gossypol Schiff's bases and optical stability of gossypolone. Eur J Med Chem 39: 619-624, 2004.

30. Sun J, Li ZM, Hu ZY, Lin XB, Zhou NN, Xian LJ, Yang DJ and Jiang WQ: ApoG2 inhibits antiapoptotic Bcl-2 family proteins and induces mitochondria-dependent apoptosis in human lymphoma U937 cells. Anticancer Drugs 19: 967-974, 2008.

31. Zhang XQ, Huang XF, Hu XB, Zhan YH, An QX, Yang SM, Xia AJ, Yi J, Chen R, Mu SJ and Wu DC: Apogossypolone, a novel inhibitor of antiapoptotic Bcl-2 family proteins, induces autophagy of PC-3 and LNCaP prostate cancer cells in vitro. Asian J Androl 12: 697-708, 2010.

32. Hu ZY, Sun J, Zhu XF, Yang D and Zeng YX: ApoG2 induces cell cycle arrest of nasopharyngeal carcinoma cells by suppressing the c-Myc signaling pathway. J Transl Med 7: 74, 2009.

33. Zhou WJ, Deng R, Zhang XY, Feng GK, Gu LQ and Zhu XF: G-quadruplex ligand SYUIQ-5 induces autophagy by telomere damage and TRF2 delocalization in cancer cells. Mol Cancer Ther 8: 3203-3213, 2009. 
34. Adams R, Morris RC, Geissman TA, et al: Structure of gossypol. XV. An interpretation of its reactions. J Am Chem Soc 60: 2193, 1938.

35. Tanida I, Ueno T and Kominami E: LC3 conjugation system in mammalian autophagy. Int J Biochem Cell Biol 36: 2503-2518, 2004.

36. Pankiv S, Clausen TH, Lamark T, Brech A, Bruun JA, Outzen H, $\varnothing v e r v a t n$ A, Bjørkøy G and Johansen T: p62/SQSTM1 binds directly to Atg8/LC3 to facilitate degradation of ubiquitinated protein aggregates by autophagy. J Biol Chem 282: 24131-24145, 2007.

37. Pyo J O, Nah J, Kim HJ, Lee HJ, Heo J and Lee H: Compensatory activation of ERK1/2 in Atg5-deficient mouse embryo fibroblasts suppresses oxidative stress-induced cell death. Autophagy 4: 315-321, 2008

38. Maiuri MC, Criollo A, Tasdemir E, Vicencio JM, Tajeddine $\mathrm{N}$ and Hickman JA: $\mathrm{BH} 3$-only proteins and $\mathrm{BH} 3$ mimetics induce autophagy by competitively disrupting the interaction between Beclin 1 and Bcl-2/ Bcl-X(L). Autophagy 3: 374-376, 2007.

39. Gao P, Bauvy C, Souquère S, Tonelli G, Liu L, Zhu Y, Qiao Z Bakula D, Proikas-Cezanne T, Pierron G, Codogno P, Chen Q and Mehrpour M: The Bcl-2 homology domain 3 mimetic gossypol induces both Beclin 1-dependent and Beclin 1-independent cytoprotective autophagy in cancer cells. J Biol Chem 285: 25570-25581, 2010.
40. Maiuri MC, Le Toumelin G, Criollo A, Rain JC, Gautier F, Juin P, Tasdemir E, Pierron G, Troulinaki K and Tavernarakis N: Functional and physical interaction between $\mathrm{Bcl}-\mathrm{X}(\mathrm{L})$ and a BH3-like domain in Beclin-1. EMBO J 26: 2527-2539, 2007.

41. Bjørkøy G, Lamark T and Brech A: p62/SQSTM1 forms protein aggregates degraded by autophagy and has a protective effect on huntingtin-induced cell death. J Cell Biol 171: 603-614, 2005

42. Boland B and Nixon RA: Neuronal macroautophagy: from development to degeneration. Mol Aspects Med 27: 503-519, 2006.

43. Lian J, Wu X, He F, Karnak D, Tang W, Meng Y, Xiang D, Ji M, Lawrence TS and Xu L: A natural $\mathrm{BH} 3$ mimetic induces autophagy in apoptosis-resistant prostate cancer via modulating Bcl-2-Beclin1 interaction at endoplasmic reticulum. Cell Death Differ 8: 60-71, 2011.

44. Pattingre S, Tassa A, Qu X, et al: Bcl-2 antiapoptotic proteins inhibit Beclin 1-dependent autophagy. Cell 122: 927-939, 2005. 